\title{
Memoria incidental e intencional en preescolares
}

\section{Miguel Angel González Francisco Valle *}

\author{
Universidad de Oviedo
}

Desde nuestro punto de vista, tres son los aspectos destacables de este artículo: r) el proporcionar una metodología - dentro del enfoque de los niveles de procesamiento-, aplicable a niños que no saben leer ni escribir (preescolares); 2) el mostrar yue la supuesta ventaja de la memoria involuntaria (aprendizaje incidental) sobre la voluntaria (aprendizaje intencional) en esta edad tal vez sea solamente aparente $y$ explicable más que por el uso de estrategias adecuadas al recuerdo, por un nivel de procesamiento profundo, en consonancia, pues, con la explicación que de la memoria adulta proporciona la teoría de los niveles de procesamiento, y 3) el ligar los niveles de procesamiento con el conocimiento previo del sujeto, aceptando como válida, en principio, la distinción entre profundidad "semántica» y profundidad "pericial» (cognitiva), en linea con las ideas de Bransford, Franks, Morris y Stein (1979).

La publicación de «Levels of processing: a framework for memory research" (Craik \& Lockhart, 1972) supuso un cambio de orientación en los estudios de la memoria que ha tenido repercusiones tanto teóricas como prácticas (pedagógicas). A nivel teórico, se ha abandonado o, al menos, se ha relegado a un segundo plano, el modelo multialmacén (MS, MCP, MLP), dominante en la década de los sesenta y se ha insistido en la profundidad del procesamiento - un proceso de control (software)-, como determinante de las caracteristicas de lo aprendido, sobre todo a nivel de recuerdo/olvido, frente a la concepción anterior en la que eran las estructuras permanentes (hardware) las determinantes de tales rasgos. En último término, la teoría de los niveles de procesamicnto ha supuesto un cambio en el concepto mismo de memoria que pierde su naturaleza (analogía) de copia o reproducción magnetofónica - esencialmente pasiva-, para convertirse en una elaboración, reconstrucción e integración de lo recién adquirido en los esquemas cognitivos previos. En el esquema anterior, la adquisición de conocimientos nuevos suponía esencialmente un incremento en el banco de datos; en el nuevo, toda adquisición supone, además, una reorganización de lo previamente conocido. Es verdad que los procesos de control no son un concepto nuevo, típico de los niveles de procesamiento. Estos habían sido tenidos en cuenta en el modelo más sofisticado del enfoque multialmacén (Atkinson \& Shiffrin, I968,

* La parte empírica de este rrabajo fue concebida y realizada, en su totalidad, por el primero de los autores. Las separatas pueden solicitarse del segundo de los autores, Departamento de Psicologia, 
1971). Pero mientras que para Atkinson y Shiffrin, el repaso es el proceso de control más importante, el que garantiza tanto la permanencia en la MCP como el aprendizaje permanente (paso a la MLP), en el nuevo enfoque se concede importancia, sobre todo, a la profundidad de procesamiento (codificación) en contra o a costa del repaso. Este último hecho ha llevado consigo una despreocupación y desprecio, tal vez exagerados, cada vez mayores del memorismo en la enseñanza y un interés creciente por lograr la comprensión --procesamiento semántico- para obtener una buena retención en los alumnos.

Anteriormente a los estudios de Craik y Tulving (1975), en los que se someten a prueba experimental algunas de las ideas teóricas de Craik y Lockhart (1972), se había mantenido que, en términos generales, los sujetos adultos recuerdan en una situación de aprendizaje incidental con tareas de orientación semántica tan bien como en una situación de aprendizaje intencional; sin embargo, los niños (Murphy \& Brown, 1975; Mújina, 1978; Marchesi, 1982, 1983) recuerdan mejor en el primer caso que en el segundo. La explicación que se ha dado de estas disparidades es que los sujetos mayores han aprendido a utilizar aquellas estrategias de memorización que producen el mejor recuerdo - procesamiento semántico-, y las usan de manera eficiente cuando se les informa que han de retener un cierto material, en tanto que los niños usarian, en todo caso, estrategias inapropiadas que, de hecho, no producen una mejora en el recuerdo (Flavell, Friedrichs \& Hoyt, 1970). Pero Craik y Tulving (1975) hallaron sustancialmente que: a) el recuerdo no depende de la intención de aprender per se, ya que el recuerdo (o reconocimiento) era aproximadamente igual, tanto si se trataba de situaciones de aprendizaje intencional como incidental; b) tampoco es función, sin más, del tiempo que se ha dedicado al problema, y c) lo que realmente determina el nivel de recuerdo es, en primer lugar, la naturaleza cualitativa del análisis que del input haga el sujeto en la codificación y, en segundo lugar, el grado de elaboración del estímulo que, dentro de cada nivel de análisis, se haya realizado. Vistas las cosas desde este punto de vista, decrece considerablemente la contraposición entre niños y adultos, ya que cuando se compara la memoria incidental con la intencional, a nivel adulto, todas las demás variables se mantienen constantes, por ejemplo, las tareas de orientación, mientras que en los estudios de memoria infantil sólo en las situaciones de aprendizaje incidental los niños han de hacer otras tareas, que exigirian un nivel de análisis profundo y que, por tanto, serian facilitadoras del recuerdo, según Craik y Tulving. Istomina (1975), trabajando con niños en edad preescolar, obtuvo un nivel de recuerdo de listas de palabras cuando esta tarea formaba parte de una actividad significativa para el niño - ir a comprar una serie de productos a una tienda para preparar la comida de sus compañeros-, dos veces mayor que cuando listas equivalentes de palabras tenían que retenerse en una situación tipica de aprendizaje de laboratorio. Resultados similares fueron obtenidos por Murphy y Brown (1975), aunque el estudio tenía finalidades distintas de las del experimento anterior. Para Mújina (1978), "la memorización involuntaria, en relación con una labor intelectual activa del niño sobre una materia determinada, sigue siendo, hasta el final de la edad preescolar, mucho más productiva que la memorización voluntaria sobre esa misma materia" (pág. 206). Pero en el caso de la memoria involuntaria, el niño tiene que distribuir las tarjetas, cuyo nombre ha de recordar, en grupos de utensilios para el 
jardín, para la cocina, etc., es decir, ha de realizar una categorización semántica, mientras que en el caso de memoria voluntaria sólo tiene que observar. Por consiguiente, puede afirmarse que los estudiosos de la memoria infantil, en su afán de buscar y encontrar diferencias entre niños y adultos, están favoreciendo el recuerdo involuntario, ya que, como se ha puesto de manifiesto en las referencias anteriores, sólo en la situación de aprendizaje incidental exigen del niño un procesamiento profundo. ¿Cuál sería el nivel de recuerdo de dos grupos de niños si la situación experimental fuera en todo idéntica, salvo que a un grupo le avisáramos de antemano que ha de recordar el material y al otro no? Desde el punto de vista de los niveles de procesamiento, lo que realmente importa es lo que el sujeto hace con la información que recibe, independientemente que sea a nivel consciente (memoria voluntaria) o inconsciente (memoria involuntaria), y ésa tal vez sea presumiblemente la explicación que se pueda dar de los resultados obtenidos con niños en tareas de memoria. El recurso al uso espontáneo de estrategias adecuadas para explicar el distinto nivel de recuerdo entre la memoria voluntaria y la involuntaria, tal vez no haga más que enmascarar el problema $o$, si se quiere, explicarío sólo a nivel superficial, en tanto que el aducir el nivel de procesamiento, como causa última, podria proporcionar una explicación única, válida tanto para preescolares como para niños mayores y adultos, y probablemente más profunda.

Por otra parte, algunas precisiones que se han hecho al enfoque de los niveles de procesamiento (Bransford, Franks, Morris \& Stein, 1979) creemos que son pertinentes para el tema que estamos considerando y también para el trabajo empírico que se va a describir. Los autores anteriores han puesto de manifiesto la relación que existe entre el material a aprender y el conocimiento y habilidades que el sujeto posee, de forma que, dado un cierto conocimiento o unos propósitos determinados, los inputs pueden ser significativos sin que sean procesados a un nivel semántico. Según estos autores, el que algo haya sido procesado a nivel profundo podría significar dos cosas diferentes, o bien que ha sido procesado a nivel semántico, o bien que el procesamiento lo ha realizado un experto o perito en la materia. Este último sentido del término ha sido recogido expresamente por Brown (1979) en su concepto de "headfitting" - que no deja de tener resonancias escolásticas-, y se encuentra de manera implícita o explícita, según los casos, en toda la obra piagetiana. De acuerdo con lo afirmado en el último párrafo, podría pensarse que para que pueda procesarse algo a un cierto nivel, es necesario suponer un banco de datos apropiado, junto con un sistema operativo adecuado y un ámbito o contexto que sea capaz de evocar tanto las operaciones como los datos necesarios. En otras palabras, las operaciones que un sujeto puede llevar a cabo (en algún sentido equivalentes a las estrategias) están intimamente ligadas a los conocimientos que ese sujeto posee y, a su vez, ambas cosas están contextualizadas. Por esta razón es sumamente interesante el trabajo de Chi (1978). En contra de la corriente dominante, esta autora ha defendido la tesis de que el menor conocimiento y uso de las estrategias, por parte de los niños, no son suficientes por sí mismos para explicar el rendimiento general menor que muestran los niños en tareas memorísticas. Sólo teniendo en cuenta, además, las diferencias de conocimiento y estructuración del mismo, se puede lograr una explicación adecuada. Este punto de vista va siendo cada vez más 
común entre los autores, por ejemplo, Huttenlocher y Burke (1976), Dempster (1977), Brown (1979), Howe y Ceci (1978), y tal vez sea posible hacer una interpretación de Piaget en este mismo sentido (Piaget \& Inhelder, 1972). Chi demostró que niños de tan solo ocho años, pero que eran buenos jugadores de ajedrez, recordaban mejor y mayor número de posiciones permitidas de fichas que adultos novicios en tal juego. Su conocimiento les permitía un procesamiento profundo "pericial" que les era imposible a adultos que no poseían tal habilidad.

El presente estudio, bastante limitado en sus fines e impreciso en algunos puntos por falta de conocimiento de la literatura sobre el tema cuando fue realizado, pretende reexaminar las diferencias en el rendimiento memorístico de niños preescolares en situaciones de aprendizaje intencional e incidental y ver el papel que el conocimiento previo del sujeto pueda tener en la memoria. Si a nivel adulto, como demostraron suficientemente Craik y Tulving (1975) lo que determina fundamentalmente el recuerdo o el reconocimiento es el nivel de procesamiento -nivel que viene determinado por la tarea de orientación-, independientemente de la intención de recordar, podría pensarse que por qué no habría de ocurrir lo mismo con los niños y que, por tanto, el nivel de recuerdo o rendimiento memoristico habría de ser semejante tanto si se trataba de memoria voluntaria como involuntaria. Aparte del hecho de que esta hipótesis encuadraba perfectamente dentro del cuadro teórico de los niveles de procesamiento, existian suficientes indicios en los autores para pensar que así fuera, ya que siempre que se hablaba de ventajas de la memoria involuntaria sobre la voluntaria, la primera siempre iba encuadrada en tareas significativas para el niño o exigía de él actividades que, desde el punto de vista de los niveles de procesamiento, serían calificadas de procesamiento semántico. Básicamente esta teoria interpreta el trazo de memoria como un subproducto del análisis perceptivo, y su cualidad y persistencia como función positiva de la profundidad de análisis. Las propias estructuras de conocimiento del sujeto son un factor determinante, ya que es a partir de ellas que el input adquiere su significación psicológica. Por eso, aun sin llegar a la posición extrema de Cascallana y Aparicio (1981) de que memorizar es conocer, pensamos que ambas cosas corren caminos paralelos (Piaget \& Inhelder, 1972) y, por tanto, el nivel de conocimiento y estructuración del mismo influirán positivamente en el recuerdo. Todos los factores que favorezcan y mejoren el análisis perceptivo darán como resultado una facilitación del recuerdo; de ahi la importancia del conocimiento del sujeto, de la motivación y de la atención.

Las hipótesis formuladas fueron las siguientes:

I. El rendimiento en una tarea de memoria con niños preescolares (tres-cinco años) es independiente de que ésta tenga carácter intencional o incidental, siempre que en ambos casos se mantengan idénticas todas las demás condiciones de la tarea.

2. Si se introduce en el material a recordar un nivel jerárquico de dificultad (entendiendo la misma como una menor adecuación a las estructuras de conocimiento del sujeto y/o a su edad), se producirá una jerarquización en el rendimiento de signo inverso a la dificultad, es decir, a mayor dificultad, menor rendimiento. 


\section{Sujetos}

Parciciparon en el experimento 23 preescolares ( 10 niñas y 13 niños) de edad comprendida entre tres y cinco años, que asistian a un mismo parvulario. En la medida de lo posible; se procuró que la representación masculina y femenina fueran semejantes en cada uno de los grupos que se formaron. Por su edad fueron clasificados en tres grupos: tres, cuatro y cinco años. El número de participantes por grupo fue el siguiente: 13 en el $1 .^{\circ}, 6$ en el $2 .^{\circ}$ y 4 en el $3 .^{\circ}$ Esta desigual proporción en cada grupo vino determinada por las condiciones del parvulario, pero creemos que no supone un problema grave, ya que precisamente el grupo más numeroso correspondió al de edad más baja.

\section{Material}

Se utilizaron dos tipos de material: objetos y dibujos. A su vez se establecieron dos grupos dentro de cada uno de ellos en función de la alta o baja probabilidad de identificación. Con ello se pretendía establecer una jerarquia en la dificultad del material de cara a su memorización, dificultad que, como ya se ha dicho, se entiende como menor adecuación a las estructuras cognoscitivas del sujeto. El orden de dificultad quedó establecido a priori de la siguiente manera:

\begin{tabular}{|l|c|c|}
\hline \multirow{2}{*}{ Objetos $\ldots \ldots \ldots \ldots \ldots \ldots \ldots$} & $\begin{array}{c}\text { Alta probabilidad } \\
\text { de identificación }\end{array}$ & $\begin{array}{c}\text { Baja probabilidad } \\
\text { de identificación }\end{array}$ \\
\cline { 2 - 3 } Dibujos $\ldots \ldots \ldots \ldots \ldots \ldots$ & Nivel I $_{\text {Nivel }}$ & Nivel 2 \\
Nivel 4
\end{tabular}

En cada nivel de dificultad se utilizaron is elementos (objetos y dibujos) que pueden verse en la figura i. En la parte de aprendizaje, a cada sujeto se le presentaban cinco elementos de cada nivel - los que le correspondían según el grupo al que hubieran sido asignadas aleatoriamente- Por tanto, en total, el número de elementos presentados fueron 20 ( 5 elementos $\times 4$ niveles). En el test de reconocimiento esos 20 elementos iban mezclados con los otros 40 distractores ( 10 de cada nivel de dificultad). Para contrabalancear el posible efecto de los elementos y del orden de presentación, se formaron ocho secuencias distintas de 20 elementos, construidas al azar en dos fases. En la primera fase se hizo un sorteo intraniveles y en la segunda interniveles. Se utilizó, asimismo, un muñeco de fabricación artesanal, dotado de mecanismo electrónico de selección y de emisión de señales luminosas y acústicas, accionado por cable desde el exterior (cf. figura 2).

\section{Situación de aprendizaje}

A la mitad de los sujetos se les informó que debían memorizar los objetos que iban viendo (aprendizaje intencional); a la otra mitad no se le proporcionó tal información. Por tanto, en el experimento se controlaron tres factores: edad, con tres niveles; tipo de material, con cuatro niveles, y situación de aprendizaje, con dos. La variable dependien- 


\begin{tabular}{|c|c|c|c|}
\hline$=0$ & $\Leftrightarrow$ & $\mathbb{1}$ & $\$$ \\
\hline$y$ & 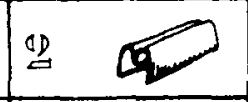 & $\therefore 585$ & घुने \\
\hline is & $\begin{array}{lll}a & \infty & 0\end{array}$ & $: B \quad 0$ & $\because \theta$ \\
\hline 11 is & 11 800 & $P_{1} \quad \&$ & \& \\
\hline$\therefore \theta$ & 吕 & 5 & 8 \\
\hline is 8 & ii $\Leftrightarrow$ & $0 \quad 8$ & a (0) \\
\hline s & $8 \quad 65$ & 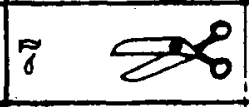 & $8 \%$ \\
\hline$\therefore$ & 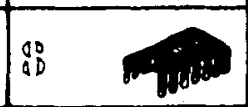 & 80 & \& 0 \\
\hline iD 8 & $\therefore$ & 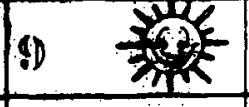 & $\$ \quad \&$ \\
\hline IOD 而而 & BOD & 10D 银 & Iid \\
\hline U1 $\$$ & 111 & 8 & 11 (8) \\
\hline ide & 埇 & 168 & 198 \\
\hline 政 & 188 8 & EBS ES & 1838 \\
\hline$i x_{1}$ & $\mathbb{R} \infty$ & If ilis & 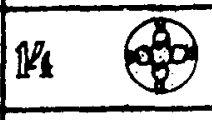 \\
\hline 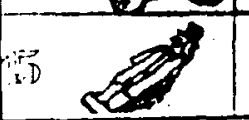 & 150 & 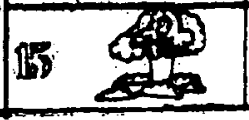 & in 8 \\
\hline
\end{tabular}

Figura I.

Elementos utilizados en el reconocimiento. En la parte de aprendizaje se usaron cinco elementos por nivel de dificultad.

te fue el nivel de reconocimiento, es decir, el número de elementos correctamente reconocidos por el sujeto. Cada identificación correcta era premiada con un caramelo que proporcionaba el muñeco. Este hecho supuso un fallo en el diseño, ya que, al no penalizarse los errores, los niños cometian muchas falsas alarmas, con lo que se aseguraban una cantidad mayor de caramelos. Por esta razón hubo que establecer un coeficiente de corrección que multiplicado por los aciertos nos daba lo que llamamos «aciertos corregidos». El coeficiente de corrección se hacía teniendo en cuenta el número de intentos necesarios para la identificación, de manera que: 


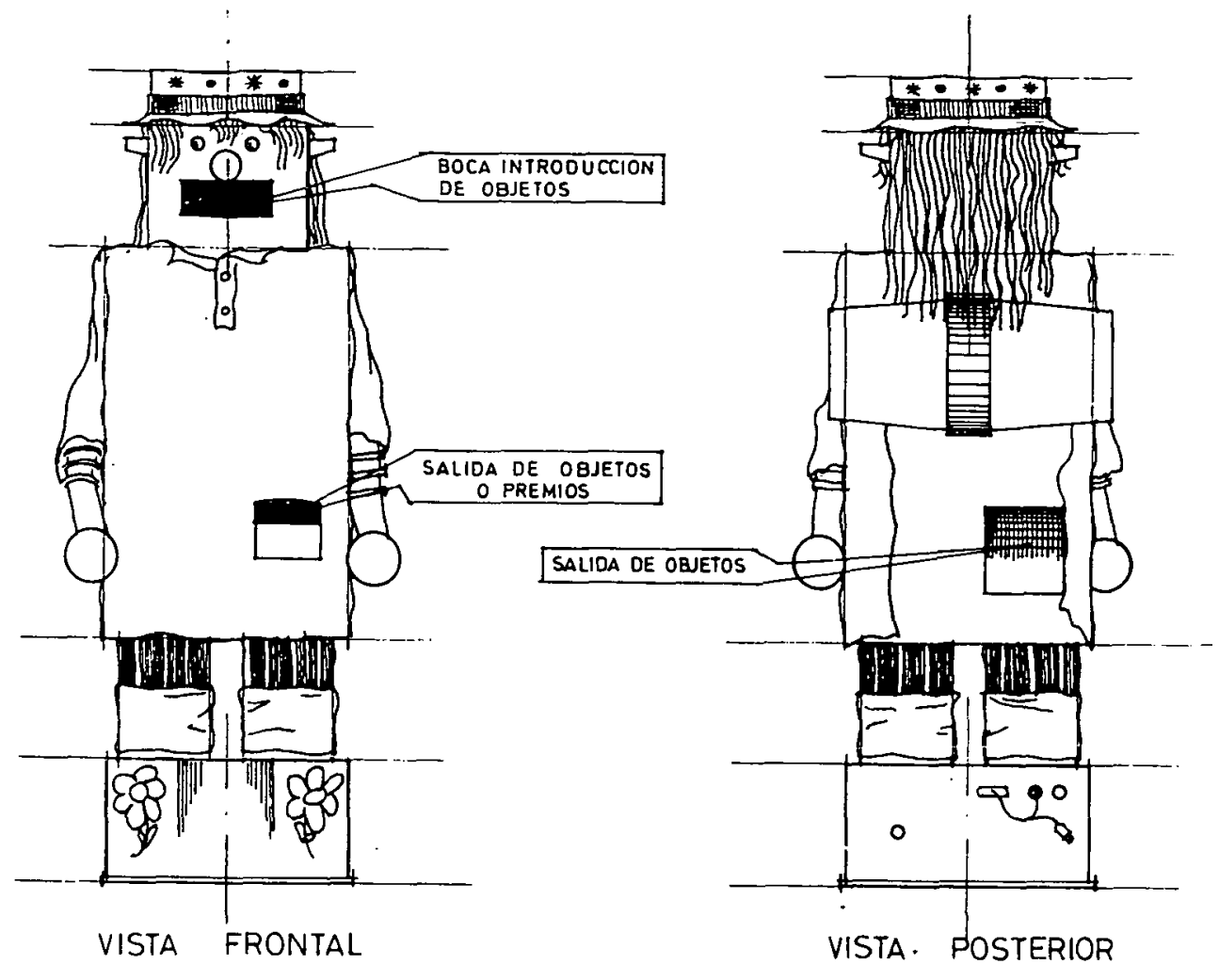

Figura 2.

Escorzo del muñeco usado.

\section{Procedimiento}

La adscripción de los sujetos se hizo de forma aleatoria (dentro de cada edad) y procurando, en la medida de lo posible, que el número de niños fuera igual al de niñas en cada condición experimental. El procedimiento general fue el mismo en todas las condiciones experimentales, salvo en lo que se refiere al apartado b) y se componia de: a) una fase inicial de familiarización con el experimento, b) instrucciones, c) presentación y manipulación del material, d) prueba de reconocimiento con premios y e) fase final de distensión.

Fase a.- Se colocó al muñeco en lugar de la clase donde todos podían verlo y se presentó por su nombre -Felipe-. Se hicieron algunos comentarios sobre su aspecto. Se les dijo que todos iban a jugar con él, pero que, como no podían hacerlo todos a la vez, cada uno lo haría por separado. Se les informó, además, de la posibilidad de ganar caramelos.

Fase b, $c y d$.- Las instrucciones fueron las mismas, salvo que a los niños de aprendizaje intencional se les advirtió que tenian que recordar los objetos - que en el juego hacían de comida de Felipe- y a los del grupo incidental no. En la prueba de memorización involuntaria se les dijo que el muñeco tenía una serie de objetos en su bolsa (estómago) y que se iba a ver de qué objetos se trataba. Para ello tendrían que meter la mano e ir sacándolos de uno en uno y diciendo su nombre en voz alta. Una vez que los había identificado los introducia en una caja 
con un orificio en su tapa, donde se encontraban el resto de los objetos (distractores de la prueba de reconocimiento). A los de la prueba de memoria voluntaria se les explicó que el muñeco tenía una bolsa con su comida, que habia que enterarse de qué se trataba, puesto que después habrian de darle de comer y sólo le gustaban los objetos que tenía antes en su bolsa (estómago). Después los iba sacando en las mismas condiciones que el grupo anterior y metiéndolos en la caja. Una vez metidos los objetos en la caja, ambos grupos tenían que ir escogiendo, después de haberlos mezclados bien con los que ya contenía la caja, del conjunto de 60 objetos aquellos 20 que había sacado del estómago de Felipe. Como ya se ha dicho antes, cada acierto era recompensado con un caramelo al tiempo que el muñco encendia sus ojos y emitía sonidos.

Fase e.-Finalmente, se procuraba cerrar la situación experimental sin brusquedad, tratando de conseguir que aquellos, que, a pesar del carácter lúdico de la prueba, hubieran padecido una situación de tensión no quedaran condicionados desfavorablemente para futuras experiencias. (Véase la figura 3, para tener una idea general de la situación experimental.)

Previamente al experimento propiamente tal se hizo una prueba piloto con tres niños de seis, siete y ocho años para comprobar la adecuación del material, del número de elementos y de si todo funcionaba bien en todos sus aspectos.

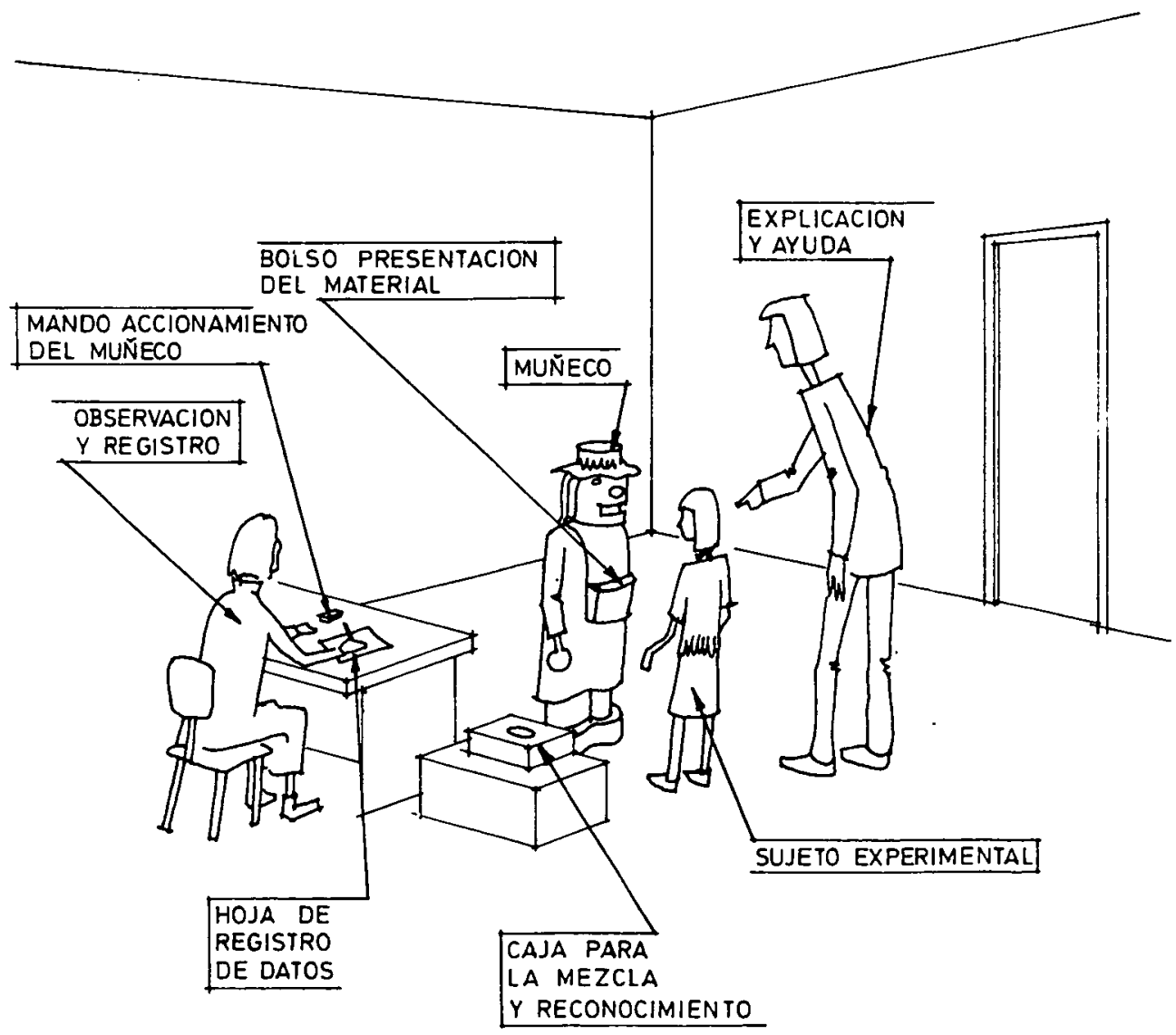




\section{RESULTADOS}

\section{Memoria voluntaria versus memoria involuntaria}

Como puede comprobarse en la Tabla i, el número de objetos y dibujos correctamente reconocidos por los niños del grupo de aprendizaje intencional fue ligeramente superior al del grupo incidental $(X=10.37, X=9.1)$, contrariamente a lo que se ha venido manteniendo. Como estadístico se utilizó la U de Mann-Whitney por tratarse de dos muestras y ser la alternativa no paramétrica (a la t de Student) más potente. El contraste estadístico mostró que no habia diferencias significativas entre los dos grupos al nivel de confianza del i por 100. No se pudo comprobar si las diferencias eran significativas al s por ıoo, por falta de tablas apropiadas, pero de cualquier forma esto no representa ninguna dificultad para el punto de vista formulado en la hipótesis primera, ya que en caso de haberla lo sería a favor del aprendizaje voluntario $y$, por consiguiente, en contra de lo que se ha mantenido previamente.

TABLA I

Promedio de Ac en las distintas condiciones experimentales

\begin{tabular}{|c|c|c|c|c|c|c|c|c|c|c|}
\hline & \multicolumn{4}{|c|}{ APRENDIZAJE INTENCIONAL } & \multicolumn{6}{|c|}{ APRENDIZAJE INCIDENTAL } \\
\hline & \multicolumn{4}{|c|}{ Nivel de dificultad } & \multicolumn{6}{|c|}{ Nivel de dificultad } \\
\hline & 1 & 2 & 3 & 4 & Total & $\mathbf{I}$ & 2 & 3 & 4 & Total \\
\hline 3 años & 2.54 & 1.93 & 3.83 & 2.5 & 10.8 & 1.75 & 1.73 & 2.44 & 1.57 & 7.49 \\
\hline 4 años & 2.89 & 1.33 & 3.46 & 1.72 & 9.4 & 1.55 & 0.97 & 2.93 & 2.93 & 8.38 \\
\hline ; años & 3.1 & 2.25 & 3.84 & 1.12 & 10.31 & 3.62 & 4.5 & 4.0 & 2.9 & 15.02 \\
\hline Media & 2.84 & 1.83 & 3.71 & 1.78 & - & 2.31 & 2.4 & 3.12 & 2.47 & - \\
\hline
\end{tabular}

\section{Dificultad del material}

En este punto se planteaban dos cuestiones; la primera, si las diferencias entre niveles eran significativas; la segunda, era la jerarquización de las diferencias. Sólo se sometió a prueba estadística la primera, por lo que se dirá más adelante. Por lo que se refiere a la primera cuestión, el contraste de Kruskall-Wallis proporcionó un $\mathrm{H}=23.88$, que para 3 grados de libertad tiene una probabilidad de ocurrencia menor de .or. Por eso se concluye que la diferencia entre los resultados de memoria en los distintos niveles de dificultad del material es significativa. Ahora bien, la jerarquización (segunda cuestión) no se correspondió con la que se habia establecido a priori. La hipótesis que se había hecho podría formularse más específicamente de esta forma: $\mathrm{Si} \quad \mathrm{DN}_{1}<\mathrm{DN}_{2}<\mathrm{DN}_{3}<\mathrm{DN}_{4}$, entonces $\mathrm{RN}_{1}>\mathrm{RN}_{2}>\mathrm{RN}_{3}>\mathrm{RN}_{4}$; donde $\mathrm{DN}=$ dificultad del nivel correspondiente, y $R N=$ rendimiento memoristico en ese nivel. Sin embargo, los datos no concuerdan con la hipótesis, ya que el promedio de elementos recordados en cada nivel fue éste:

$$
R N_{1}=2.39, R_{2}=1.92, R_{3}=3.31, R_{4}=2.12
$$


Esto parece indicar que la diferencia entre objetos y dibujos no es la esperada. De hecho a lo largo de las ruebas se pudo observar un mayor interés por las fichas (dibujos) que por los objetos, lo cual quedó, además, demostrado el fin final de la prueba en el que se hizo una pequeña fiesta. Se colocó todo el material -objetos y fichas- en el suelo y se pidió a los niños que eligieran, uno por uno, el objeto o ficha que más le hubiese gustado. El resultado, si bien no tiene pretensiones de rigor, se incluye aquí por su revelancia para el punto que estamos comentando. El número de elecciones que obtuvo cada nivel, indicador de algún modo de su atractivo para los niños, puede verse en la Tabla 2.

TABL.A 2

Relacion entre interés por el material de recuerdo y promedio de elementos reconocidos

\begin{tabular}{lrrrr} 
& \multicolumn{1}{c}{} & $\mathrm{N} .0$ de eleceiones & $\mathrm{X}$ de reconocimiento & Rango \\
\hline Nivel 1 & $\ldots \ldots \ldots$ & 9 & 2.39 & 2 \\
Nivel 2 & $\ldots \ldots \ldots$ & 3 & 1.92 & 4 \\
Nivel & $\ldots \ldots \ldots$ & 11 & 3.31 & 1 \\
Nivel & $\ldots \ldots \ldots$ & 4 & 2.12 & 3 \\
\hline
\end{tabular}

Si utilizáramos ahora esta clasificación como índice de dificultad del material, entonces se observaria que el nivel de reconocimiento de cada grado de dificultad guarda un estrecho paralelismo con el interés manifestado por los elementos componentes de esos niveles. Además, este orden de preferencias es fundamentalmente el mismo tanto si se trata del grupo de memoria incidental como del internacional.

Edad.-En cuanto a la edad, la $\mathrm{H}$. de Krushall-Wallis fue 45.69 que para dos g.l. tiene una probabilidad menor de 0.01 , lo que indica que hay diferencias significativas entre los grupos, pero el bajo número de sujetos en alguno de los grupos de edad hace aconsejable no conceder demasiada importancia a los resultados hasta no haber sido confirmados con una muestra más representativa. No obstante, conviene señalar que el efecto de la edad queda limitado exclusivamente al grupo de aprendizaje incidental que es donde aparecen diferencias notables en lo que se refiere al nivel de reconocimiento, según la edad. En el grupo de memoria voluntaria, por el contrario, las diferencias de recuerdo entre los diferentes grupos de edad son prácticamente nulas.

\section{DISCUSION}

En primer lugar conviene señalar que somos conscientes de las imperfecciones del trabajo; unas debidas a circunstancias ajenas a nuestra voluntad, como por ejemplo, el hecho de que de los 30 niños con que se había pensado trabajar en un principio nos fallara, por unas razones o por otras, una proporción considerable ${ }^{7}$; otras de las que somos completamente responsables, como el no haber revisado la literatura sobre el tema - una parte importante de la misma - antes de diseñar el experimento, lo cual hizo que algunas partes del mismo resultaran pobres, como la que se refiere a la hipótesis segunda, y que 232 podrian haber sido mucho más productivas y haber arrojado más luz 
sobre los mecanismos de la memoria y su interrelación con el conocimiento, de haber conocido, entre otros, los trabajos de Chi (r978) o Markman (1977). Pero, por otra parte, el haberla revisado a posteriori ha puesto de manifiesto que las ideas de las que partíamos eran en principio coincidentes con las de autores significativos dentro del campo de la memoria infantil. Pero a pesar de sus limitaciones conviene destacar algunas de las aportaciones del presente artículo. En primer lugar, ha puesto de manifiesto que no existe discontinuidad -al menos a nivel explicativo- entre los resultados de la memoria con adultos y con niños en edad preescolar, ya que en ambos casos, como había señalado la teoria de los niveles de procesamiento, lo único importante o al menos lo fundamental, es lo que el sujeto hace con la información que se le presenta y que nunca es un factor decisivo la intención de retener per se (o la falta de la misma). Los estudios previos en los que siempre se señalaba las ventajas de la situación incidental sobre la intencional, en el fondo estaban confundiendo dos variables: el nivel de procesamiento y el carácter voluntario/involuntario. Esta confusión era imposible de detectar al ligar siempre la situación de aprendizaje incidental con tareas significativas para el niño o que exigían categorizaciones, análisis discriminativos, etc., funciones que, desde el punto de vista de la profundidad de procesamiento obligarían al niño a un procesamiento profundo (semántico). Cuando estas dos variables se han separado, se ha visto que tampoco aquí es la intencionalidad algo fundamental. $Y$, por consiguiente, no se puede seguir manteniendo, al menos sin matizar, la opinión generalizada y que resumimos aquí citando textualmente a Harris (1978): «Aunque el niño pequeño no usa con frecuencia estrategias en las llamadas tareas de memoria intencional, sin duda obtiene buenos resultados en ciertas tareas en las que parece que no se ha de ejercitar un esfuerzo mnemónico deliberado" (pág. 372). Sin embargo, los defensores de la opinión anterior pueden seguir diciendo que al menos existe una diferencia entre adultos y pequeños, porque los primeros pueden ejercitar niveles de procesamiento adecuados para un buen recuerdo, aun en situaciones de aprendizaje intencional "puro», es decir, sin tarea de orientación semántica, mientras que los niños son incapaces. $Y$ aun admitiendo la verdad de la afirmación anterior, creemos que lo único que se puede concluir es que el decir a un niño que aprenda una lista de palabras no es suficiente para desencadenar análisis perceptivos adecuados, que tal recomendación no tiene o no ha adquirido la significación social o vital que puede tener para los adultos. Tal vez lo que se quiera decir con esto es que los mecanismos de la memoria, por ejemplo, el procesar a un nivel profundo se desencadenen automáticamente con tal que la situación tenga un significado para el sujeto, sea del tipo que sea. (Esto con todo es una simple conjetura, sin suficientes datos empíricos.)

Creemos que también ha sido interesante, en cuanto posibilitadora de experiencias posteriores, la metodologia empleada. Para muchos el marco teórico de los niveles de procesamiento, aunque interesante y fructífero en sí mismo, se veía limitado en su aplicación, ya que utilizar material verbal presentado visualmente exigía un cierto nivel de escolarización en los sujetos. Al usar material visual (pero no palabras), junto con una tarea mucho más divertida - jugar a tratar de averiguar qué come un muñeco- hemos hecho posible la aplicación de la metodología de los niveles de procesamiento a una población para la que, en principio, no fue pensada, dadas sus características. 
En lo que se refiere a la jerarquía del material y a la comparación entre el rendimiento memorístico en las distintas edades, que era el objetivo secundario del trabajo, podemos decir que si bien los resultados no confirman las hipótesis tal y como se enunciaron, si se han encontrado algunas cosas interesantes. En primer lugar, no se recuerda igual un material que otro; hay diferencias en el rendimiento. Esto sí se ha probado estadísticamente. Lo que no se confirmó fue la jerarquización supuesta, pero sí apareció una jerarquía en los rendimientos, cuyo orden coincidía exactamente con las preferencias subjetivas de los niños, y que en consecuencia deben estar interrelacionadas. Si así es habria que suponer que el atractivo del material es también una de las condiciones facilitadoras del recuerdo, lo cual por otra parte no constituye ninguna novedad; pero tal vez el intentar dar una explicación de por qué esto ocurre obligaría a un replanteamiento total incluso de la teoria más actual de la memoria, o sea, de los niveles de procesamiento.

\section{Referencias}

AtKinson, R. C., y SHIFFRIN, R. M.: "Human memory: a proposed system and its control processes." En K. S. SPENCE y J. T. SPENCE (Eds.): The Psychology of Learning and motivation: Advances in Research and Theory, Vol. 2, Academic Press, 1968.

Atkinson, R. C., y SHiffrin, R. M.: "The control of short-term memory". Scientific American, 224, págs. 82-89. 1971 .

Bransford, J.; Franks, J.; Morris, C. D., y STEIN, B. S.: "Some general constraints on learning and memory research». En L. S. Cermak y F. I. M. Craik (Eds.), Leyels of Processing in Human Memory, LEA, 1979 .

BRown, A. L.: “Theories of memory and the problems of development: activity, growth and knowledgem. En L. S. Cermak y F. I. M. Craik (Eds.), O. C.

Cascallana, M., y Aparicio, J. J.: «Memorizar es conocer: un estudio sobre la influencia de la información previa en las tareas de recuerdo. Infancia y Aprendizaje, J6, págs. 17-27. 1981.

CHI, M.: «Knowledge structures and memory development». En R. S. SIEGLER (Ed.), Children's Thinking. What Develops?, LEA, 1978.

CRAIK, F. I. M., y LOCKHART, R.: "Levels of processing: a framework for memory research». Journal of Verbal Learning and Verbal Behavior, 11 , págs. 671-684. 1972. (Trad. cast. en Estudios de Psicologia, núm. 2).

CRAiK, F. I. M., y TULVING, E.: "Depth of processing and the retention of words in episodic memory". Journal of Experimental Psychology General, 104, págs. 268-294. 1975. (Trad. cast. en Estudios de Psicologia, núm. 2).

Dempster F. N.: Short-term Storage Capacity and Chunking: A Developmental Study. Tesis doctoral, Universidad de California, 1977.

Flaveli, J.; Friedrichs, A., y HoYt, J. D.: "Developmental changes in memorization processes». Cognitive Psychology, I, págs. 324-340. 1970.

Harris, P. L.: "Developmental aspects of memory: a review». En M. M. GruneberG, P. E. Morris, y R. N. Sykes (Eds.), Practical Aspects of Memory. Academic Press, 1978.

Howe, M. J. A., y CECI, J. J.: "Why older children remember more: contributions of strategies and existing knowledge to developmental changes in memorym. En M. M. GrUneberG, P. E. MORRIS, y R. N. SYKES (Eds.), o. c.

HUtTEnlocher, J., y BURKe, D.: «Why does memory span increase with age» Cognitive Psychology, 8, págs. I-31. 1976.

Istomins, Z. M.: "The development of voluntary memory in pre-school children». Soviet Psychology, 13, págs. 5-64. 1975 .

MARCHESI, A.: «El desarrollo del conocimiento sobre los propios procesos cognitivos. Anotaciones acerca del interés sobre el sujeto en psicología evolutivan. En El sujeto en la Psicologia Cientifica Actual, $70^{\circ}$ Congreso Nacional de Psicología, Santiago, 1982.

MARCHESI, A.: «La memoria de los niños». Cuadernos de Pedagogia, 97, págs. \$3-59. 1983.

MARKMAN, E. M.: «Realizing that you don't understand: a preliminary investigation». Child Development, 48, págs. 986-992. 1977.

MújINA, V.: Psicologia de la edad preescolar. Pabló del Rio, 1978.

MURPHY, M. D., y BRown, A. L.: "Incidental learning in pre-school children as a function of level of cognitive analysis": Journal of Experimental Child Psychology, 19, págs. 509-523. 1975. 


\section{Resumen}

En general, se ho mantenido que los niños en edad preescolar recuerdan mejor en tareas de aprendizaje incidental que en las de aprendizaje intencional. En este estudio se ha reexaminado esta cuestion y se bo visto que no existe tal diferencia. Niños de ; as reconocieron de modo semejante une serie de objetos y dibujos, tanto si se les habia advertido previamente que debian recordar como si no. El estudio ba demostrado asimismo, y en concordancia con otros trabajos, que el recuerdo no es independiente del nivel de conocimiento ni de la edad del niño. En virtud de estos resultados se bace una interpretación de la memoria infantil acorde con la teoria de los niveles de procesamiento.

\section{Summary}

It has been defended that, in general, pre-school children recall in incidental learning better than in intentional learning tasks. This issue has been reexamined in this study and no statistical difference bas been found. No matter whether or not they were told in advance that they would have to retain the material, 3 - to s-year old children recognized a series of objects and drawings with equivalent accuracy. The experiment bas also shown, in agreement with previous studies, that recognition is not independent of the children's level of knowledge (or age). Based on these results, an interpretation of children's memory is made from the levels of processing framework.

\section{Résumé}

On ité defendu que les enfants à läge prescolaire rappellent mieux quand ils n'ont pas été avertis de retenir que quand ils ont refu cette information prialablement. Cette question a été rexaminée et aucune différence statistique a ité trouvée. En un test de reconnaissance d'objects et dessins, enfants de 3 à ans, ont obtenu de résultats mnésiques semblables dans une situation d'apprentisage incidental et dans une autre intentionelle. L'etude a montré aussi que la memoire n'est - pas independente du niveau de connaissance ni de l'äge de l'enfant: D'après ces résultats, on fait une interpretation de la memoire enfantine d'accord avec la théorie des niveaux de processus. 